\title{
Zweiter Teil: Die ökonomische und technische Perspektive
}

Für Wirtschaft und Technik stellt sich manches ganz anders dar als für Staat und Politik. Während die öffentliche Verwaltung an Recht und Gesetz gebunden ist, ja ohne gesetzliche Grundlage gar nicht eingreifend tätig werden darf, sind unternehmerische Initiative und richtiger Einsatz der Technik die wichtigsten Faktoren wirtschaftlichen Erfolges; wer etwas unternimmt, nutzt seine Freiheit und darf darin nur beschränkt werden, wenn dies verfassungsrechtlich zugelassen ist. Das Internet zu nutzen ist in dieser Perspektive ebenfalls ein Akt der Freiheit, aber das Recht ist auch hier im Spiel - als die Instanz, die Handlungsräume eröffnet und gleichzeitig Grenzen zieht.

\section{Geschäftsmodelle und Risiken}

Die Internetwirtschaft boomt. Das Netz und seine Umgebung sind für Unternehmer eine Goldgrube. Allein der ständige Ausbau und die Unterhaltung des Netzes erfordern große Investitionen und bieten Renditechancen. In besonders großem Maßstab wird an den angeschlossenen Geräten und den notwendigen Programmen verdient. Von dem gewaltigen Stromverbrauch für das Internet und die daran teilhabenden Computer wird zwar selten gesprochen; es liegt auf der Hand, dass auch dieser Aufwand die Wirtschaftsleistung der beteiligten Länder erhöht. Dass im Netz und um das Netz herum unzählige Kauf- und Dienstleistungsangebote platziert werden, bedeutet eine riesige Ausdehnung der Märkte.

Informationen werden bisher vielfach unentgeltlich angeboten, aber die Netzbetreiber lassen sich die entsprechende Werbung von den Anbietern bezahlen. Die Nutzer wiederum ,zahlen“ - meist ohne sich das klarzumachen - mit ihren persönlichen Angaben; die Daten sind im Internet eine wertvolle „Währung“. ${ }^{153}$ Vielen halten diesen ,freien“ Markt für den Normalzustand und die Entgeltlichkeit von Medienangeboten für die unerwünschte Ausnahme - aber das widerspricht der Logik der Wirtschaft. Nur solange die Kosten der Webseiten durch die Werbeeinnahmen überkompensiert werden, kann es bei der Unentgeltlichkeit bleiben; diese Zeiten werden vielleicht schon bald vorbei sein.

Niemand weiß, welche Firmen dauerhaft Gewinne bringen werden. In den wenigen Jahren, seit es das Internet gibt, sind bereits einige Unternehmen abgestürzt, waren plötzlich nur noch einen Bruchteil dessen wert, was die Börse, die Rating- 
agenturen oder die Fachpresse ihnen attestiert hatte; viele, die mit großen Erwartungen gestartet waren, sind nach einiger Zeit wieder ganz von der Oberfläche verschwunden. In keinem anderen Wirtschaftszweig waren die „Blasen“ so groß und sind so spektakulär geplatzt wie in der Informations-, Medien- und Kommunikationswirtschaft. Die „sozialen Netzwerke“ wie Facebook und Twitter leben von aktuellen sozialen Moden. Sie sprechen vor allem die Jugend an (und in ihrem Gefolge alle möglichen Institutionen und Unternehmen, die sich unter dem Titel „Freunde“ neue Kunden oder Sympathisanten beschaffen wollen). Wenn die jugendlichen Facebook-Enthusiasten sich an die neuen „Freundeskreise“ gewöhnt haben, finden sie es vielleicht bald gar nicht mehr ,cool“, sondern langweilig, ständig dort zu kommunizieren. Sie werden älter und wollen vielleicht von all dem schönen Zauber gar nichts mehr wissen. Dann kann es sehr schnell mit den Teilnehmerzahlen abwärts gehen. Sind die Unternehmen dann hoch verschuldet oder leisten sie sich Managementfehler, ist ihre Existenz gefährdet. Andere Unternehmen werden auf ihren Spuren wandeln und versuchen, die Märkte auszuschöpfen, aber möglich ist auch eine allgemeine Erschöpfung der Internet-Beziehungen und eine Rückkehr zu anderen Formen sozialen Kontakts. Die vorübergehende Schwäche der Facebook-Aktien an den Börsen war ein Warnsignal: Die Bäume wachsen nicht in den Himmel.

Kurz gesagt, ist die Lage der Internetwirtschaft nicht besser und nicht schlechter als die anderer Wirtschaftszweige - nur dass die finanziellen Volumina, um die es geht, oft erheblich größer und die Entwicklungsaussichten ungewisser sind. Die Online-Wirtschaft kann in kurzer Zeit mit relativ geringem Aufwand so große Mengen von Individuen als Kunden gewinnen wie kein anderer Wirtschaftszweig; daher sind die Gewinnchancen so groß wie kaum irgendwo sonst. Ähnlich groß ist aber auch das Verlustrisiko. Im Kern aber gilt: Ob online oder offline, die Entwicklung eines Unternehmens hängt mehr von den Geschäftsideen und den Personen an der Spitze ab als von der technischen oder nicht-technischen Vermittlung der Angebote.

In der Netzwirtschaft hat sich ursprünglich eine Gruppe klarsichtiger junger Leute durchgesetzt, die frühzeitig das enorme wirtschaftliche Entwicklungspotential technischer Geräte und technisch vermittelter Leistungen erkannten und die den Mut hatten, mit wenig Eigengeld und viel Schulden, aber auch mit großer Disziplin und persönlichem Einsatz unternehmerisch anzufangen. Sie trafen den Geist der Zeit, und da „nichts erfolgreicher ist als der Erfolg“, sind sie von Beobachtern und Begleitern immer höher gejubelt und geschrieben worden. Je mehr Menschen sich Computer kaufen und im Netz surfen, desto glänzender strahlt der Ruhm der Erfinder und Manager. Um Ruhm und Größe aber rankt sich Geheimnis, Kritik verstummt, Phantasie blüht. 
Erwartbar war auch, dass die Ausdehnung der Internetwirtschaft einige bisher erfolgreiche Geschäftsmodelle in Bedrängnis gebracht hat. Wenn sehr viele Menschen sich im Internet orientieren, dort Bestellungen aufgeben und sich Musik anhören und Videos ansehen, gehen die Umsätze der traditionellen Medien, des Buchhandels und der Platten- und Filmhersteller zurück. „Einige der wichtigsten Sub- und Popkulturphänomene" sind heute im Netz zu finden ${ }^{154}$. Die bisher auf diesen Märkten etablierten Unternehmen müssen sich umstellen: Die Hersteller von Lexika bedienen die Leserschaft ebenso über das Netz wie die Zeitungen und Fernsehanstalten ihr elektronisches Angebot durch online-Angebote ergänzen.

Der Streit um das Urheberrecht: Das Ob und das Wie

\section{Die Gewöhnung an Unentgeltlichkeit}

Viele meinen nun, dass auch die Nutzung des Netzes und der dort angebotenen Informationen und Kommunikationsmöglichkeiten für jedermann unentgeltlich sein müsse. Das ist aber alles andere als selbstverständlich. Unser Wirtschaftssystem beruht vielmehr darauf, dass jede Leistung - zumindest jede individuell zurechenbare Leistung - eine Gegenleistung verlangt. Nur ausnahmsweise erbringt die Allgemeinheit die Gegenleistung oder der Leistende finanziert sie auf andere Weise, im Netz vornehmlich durch Einnahmen aus der Werbung und der Auswertung von Kundendaten. Die Theorie der „Allmende“, also der genossenschaftlichen Nutzung von Gemeingütern unabhängig von Marktmechanismen, ${ }^{155}$ findet zwar neuerdings unter Netzexperten Aufmerksamkeit, bleibt in der Praxis der Wirtschaft aber bislang ohne Wirkung.

Die Erwartung der meisten Nutzer, dass im Netz kostenlos zu haben sein müsse, was offline seinen Preis hat, beruht offensichtlich auf Gewohnheit, nämlich auf der Erfahrung, dass eben dies bisher möglich ist. Ist aber dadurch schon Gewohnheitsrecht entstanden? Wir sind durch die unentgeltliche Präsentation der Inhalte von Zeitungen und Zeitschriften und durch das Herunterladen aller möglichen Programme verwöhnt, ohne viel darüber nachzudenken, wie denn die Kosten des Netzes aufgebracht werden. ${ }^{156}$ Viele finden es auch ganz in Ordnung, dass sie Musik und Filme, die sie sonst kaufen oder gegen Entgelt ausleihen müssten, ,umsonst“ aus dem Netz beziehen - womit meist Urheber- und Leistungsschutzrechte der Autoren, Komponisten und darbietenden Künstler verletzt werden. Seit einiger Zeit lassen die Rechteinhaber solche illegalen Nutzungen durch Verwertungsgesell-

154 Kreye 2011.

155 Vgl. Ostrom 1999.

156 Wie hoch diese sind, haben u.a. Kurz/Rieger 2012, S. 14 ff. anschaulich beschrieben. 
schaften oder Anwälte systematisch verfolgen, kassieren zunehmend Entgelte und verlangen - mit Erfolg - auch Erstattung der Gebühren für diese Rechtsdurchsetzung; einige Anwaltsbüros verdienen prächtig an dem formularmäßigen Einzug dieser Gelder. ${ }^{157}$ Durch Fehler bei der IP-Feststellung werden dabei offenbar immer wieder auch die Falschen herangezogen, und angesichts der allgemeinen Technikgläubigkeit fällt es ihnen schwer zu beweisen, dass sie die betreffenden Inhalte gerade nicht heruntergeladen haben.

Kein Wunder, dass die ertappten Sünder und eben auch rechtmäßige Internetnutzer die „Freiheit des Internet“ beschwören; niemand lässt sich gern rechtswidriges Handeln vorwerfen, und niemand zahlt gern die Kosten seiner eigenen Demütigung. Zur Entschuldigung der Urheberrechtsverletzer mag es auch beitragen, dass viele Internet-Anbieter in der Vergangenheit eben nicht auf Entgelten bestanden haben, sondern die kostenlosen Angebote als verlockende Kostproben ihrer Produktion, also zu Werbezwecken ins Netz gestellt haben.

Dass das Surfen im Netz frei sein soll, folgt - wie ausgeführt - aus der grundrechtlichen Informationsfreiheit gemäß Art. 5 Abs. 1 Satz 1, Halbsatz 2 GG: Das Netz ist eine ,allgemein zugängliche“ Informationsquelle. Aber das Grundrecht garantiert nicht die unentgeltliche Teilhabe an dem im Netz gespeicherten Wissen. Den Anbietern steht es frei, ein Entgelt zu verlangen: Dass eine Zeitung am Kiosk ihren Preis hat, stört niemanden - dasselbe Produkt, der leichteren Zugänglichkeit halber ins Netz gestellt, braucht nicht verschenkt zu werden. Der Markt verlangt möglicherweise andere Modalitäten der Bezahlung, aber er lässt sich nicht zum Verschenken von Leistungen verleiten, und der Gesetzgeber oder die Gerichte haben keinen Anlass, die Unentgeltlichkeit zu erzwingen.

\section{Der Kampf um die Rechtspositionen}

Der Streit um das Urheberrecht wird gegenwärtig weltweit ausgetragen. In den USA hat man sich insbesondere über zwei Gesetze gestritten, die der Internet„Piraterie“ entgegenwirken sollen: der Stop Online Privacy Act, kurz Sopa genannt, und der Protect IP Act, Pipa. Darin ist u.a. vorgesehen, dass Seiten aus den Suchmaschinen genommen und IP-Adressen gestrichen werden. Die Internet-Firmen wie Google, Facebook, Twitter und YouTube „laufen Sturm“ gegen diese Initiativen. ${ }^{158}$ Sie rufen „Zensur" und behaupten, die Meinungsfreiheit der Intenetnutzer zu verteidigen. Auf der anderen Seite aber kämpfen die großen Medienunternehmen wie Time Warner, Sony und CBS für die Rechteinhaber und ihre eigenen Vermarktungsinteressen, dazu die Lobbyorganisation der amerikanischen

157 Krit. dazu mit Grund u.a. Kurz 2012.

158 Klüver 2012. 
Filmwirtschaft, die US-Handelskammer und der Gewerkschafts-Dachverband AFL-CIO, die ebenso wie ihre Gegenspieler um ihre Geschäfte und die Arbeitsplätze bangen. Es bleibt nicht mehr verborgen, ,dass hinter den Anti-Zensur-Parolen handfeste wirtschaftliche Interesse stehen". 159

Internet-Aktivisten haben auch in Deutschland mit großem Aufwand und scharfen Worten erfolgreich die Ratifikation des multilateralen Anti-Counterfeiting Trade Agreement (ACTA) bekämpft, durch das neue internationale Standards für die Abwehr von Produktpiraterie und Urheberrechtsverletzungen verbindlich werden sollten. ${ }^{160}$ Dieses „Anti-Piraterie-Abkommen“ hatten die USA, Kanada, Japan, Australien, Neuseeland, Südkorea, die Schweiz sowie die Europäische Union und die meisten ihrer Mitgliedstaaten ausgehandelt. Die Staaten sollten danach auf diesem Feld effektiver kooperieren, und sie sollten ausdrücklich verpflichtet sein, die Rechte der Urheber durchzusetzen. Gegen einige der Maßnahmen, die in dem Abkommen als „geeignet“ bezeichnet wurden, richteten sich wütende Proteste, vor allem gegen Netzsperren und dagegen, dass Internet-Dienstleister auch für inhaltlich rechtswidrige Internet-Veröffentlichungen verantwortlich sein sollen. ${ }^{161}$ Ihre rabiaten Methoden (wie die Hackerangriffe auf Webseiten der Regierungen und anderer ACTA-Befürworter) versuchte die „Web-Guerilla“damit zu rechtfertigen, dass die Verhandlungen lange geheim geführt worden sind und dass keine Vertreter der Internetnutzer hinzugezogen worden seien ${ }^{162}$ - fast so als ob die Interessenten ein eigenes Volk bildeten, mit dem die staatlichen und internationalen Instanzen auf gleicher Ebene verhandeln müssten.

Nachdem nun auch die amerikanische Justiz gegen die Datentransferbörse $\mathrm{Me}$ gaupload vorgegangen ist, die ihre Nutzer für die Bereitstellung rechtswidriger Kopien belohnte, haben Hacker der Gruppe „Anonymous“ in einem angekündigten „Rachefeldzug“" vorübergehend die Webseiten des FBI und des US-Justizministeriums lahmgelegt. ${ }^{163}$ Tauschplattformen sind für ihre Betreiber offensichtlich ein gutes Geschäft; darum wird mit harten Bandagen gefochten.

Die Kritiker haben sich auf die höchsten Normen der ungeschriebenen oder geschriebenen Weltrechtsordnung berufen. So hat amnesty international behauptet, ACTA sei „eine Büchse der Pandora für mögliche Menschenrechtsverletzungen“; es habe „negative Auswirkungen auf mehrere Menschenrechte, insbesondere das Recht auf ein angemessenes Verfahren, das Recht auf Achtung des Privatlebens, die Informationsfreiheit, Meinungsfreiheit und das Recht auf Zugang zu lebens-

159 Koch 2012

160 Text: Rat der Europäischen Union, Dokument 12196/3/11 REV 3 (de), im Internet leicht zugänglich.

161 Vgl. nur Brill 2012.

162 Brill aaO.

163 Koch/Brinkmann 2012. 
wichtigen Medikamenten“"164, und wikipedia überschreibt den entsprechenden Abschnitt seines ACTA-Beitrags mit „Aushebelung der Menschenrechte und des Rechtsstaates" - als ob das eine Tatsache wäre. Wenn das zuträfe, könnte man sich einen größeren Skandal kaum vorstellen - das Abkommen hätte dann gleiches Gewicht und wäre ungefähr ebenso verwerflich wie die Aufhebung der UN-Menschenrechts-Erklärung oder die weltweite Abschaffung der Pressefreiheit. Erstaunlich, dass solch eine offensichtliche Übersteigerung die vielen Menschen, die sich betroffen fühlen, nicht misstrauisch macht.

Entgegen dem, was die weltweite Kampagne gegen den ACTA-Entwurf behauptet, sind darin keineswegs bestimmte Maßnahmen vorgeschrieben, sondern es handelt sich eben nur um eine Aufforderung an die Vertragsstaaten, Durchsetzungsverfahren bereitzustellen. Und das Recht auf ein angemessenes Verfahren wird nicht beeinträchtigt, sondern es wird ausdrücklich gesagt, dass die entsprechenden Maßnahmen „fair" und ,gerecht" sein und in „,angemessenem“Verhältnis zu der Schwere der Rechtsverletzung und den Interessen Dritter stehen müssen. ${ }^{165}$ Viele der empfohlenen Instrumente sind schon Teil des deutschen Rechts. Geht es manchen Organisatoren der Kritik vielleicht doch in erster Linie um den Erhalt der wirtschaftlichen Vorteile, die sie aus der Verletzung von Urheber-, Marken- und verwandten Schutzrechten ziehen? Wenn eine Bürgerrechtsorganisation sich auf eine so maßlose Propaganda einlässt, macht sie sich unglaubwürdig; bei aller Kritik an der Ökonomisierung aller Lebensverhältnisse könnte sie wissen, dass die Menschenrechte nicht gerade durch ein solches rechtstechnisches Abkommen gefährdet sind.

Richtig ist freilich, was amnesty international anmerkt, dass nämlich die strengere Durchsetzung von Urheberrechten die Internetdiensteanbieter zu „repressiven Maßnahmen" wie dem Sperren oder Löschen von Webseiten oder gar zum Ausschluss der Kunden von Dienstleistungen anreizen würde. Aber wäre es nicht genau richtig, wenn die Internetunternehmen - im Rahmen ihrer Erkenntnismöglichkeiten! - selbst darauf achten müssten, dass sie nicht zur Verletzung fremder Rechte beitragen? Es mag nicht immer leicht sein, insofern sichere Erkenntnisse zu erlangen, und selbstverständlich könnte über strittige Fälle vor den Gerichten gestritten werden. Wenn denn aber feststeht, dass angebotene Inhalte nicht verbreitet werden dürfen, können sich Anbieter wie Nutzer nicht auf Informationsoder Meinungsfreiheit berufen. ${ }^{166}$ Beim Handel mit gestohlenen Büchern oder CDs würden wir von Hehlerei sprechen, und niemand würde sagen, die Händler müssten um der Meinungsfreiheit willen ermächtigt werden, die Augen fest zu schließen

164 Erklärung von amnesty international vom 13.2.2012, www.amnesty.de/2012/2/14/eu-darfacta-nicht-unterzeichnen.

165 Art. 6 des Entwurfs.

166 S. a. Prantl 2012 mit der Klarstellung, dass das Urheberrecht ja nicht Informationen als solche, sondern „Werke“, also gestaltete Texte usw. schützt. 
und alles als ehrliche Ware anzusehen. - Eine ganz andere Problematik wirft der Hinweis von amnesty auf die Versorgung mit Medikamenten auf: Würden Generika generell als Erzeugnisse von „Produktpiraterie“ verstanden, so wäre das mit unserer Gesundheitspolitik unvereinbar. Das hat aber mit dem Streit um ACTA kaum etwas zu tun.

Eine Gruppe von Professoren aus verschiedenen Staaten - überwiegend aus Deutschland - hat in einer wohlabgewogenen Erklärung bemängelt, dass bestimmte Vorschriften in ACTA nicht mit dem geltenden europäischen Gemeinschaftsrecht vereinbar seien und/oder hinter den Standards des internationalen Rechts zurückblieben. ${ }^{167}$ So sicherten einige ACTA-Regelungen keine ausreichende Abwägung zwischen den Interessen der Beteiligten oder es seien keine Maßnahmen vorgesehen, um die Durchsetzung der Vorbehalte und die Einhaltung der Grenzen der Befugnisse zu gewährleisten. Nötig seien z.B. spezifischere Vorschriften darüber, wie die Rechte auf Meinungsfreiheit, faires Verfahren und Privatheit effektiv gesichert werden sollen. Das sind rechtstechnische Details, die eher geringes Gewicht haben - zumal sich die Unterzeichner grundsätzlich zum Schutz der Rechte des geistigen Eigentums bekennen. Es ist ja durchaus wünschenswert, die Rechtslage aller Beteiligten möglichst eindeutig zu bestimmen, aber wo das Recht nur auf Prinzipien verweist, kann daraus nicht die Lösung aller denkbaren praktischen Fälle abgeleitet werden. Es unterhöhlt letztlich sogar die Geltungskraft von Rechtsvorschriften, wenn ihre Durchsetzung jeweils speziell vorgeschrieben wird - dass sie befolgt werden, ist selbstverständlich, und Selbstverständliches sollte nicht noch einmal normiert werden. Die zahllosen Absicherungswünsche signalisieren wohl nur, dass man der anderen Seite grundsätzlich misstraut. Solange das so ist, werden immer aufs Neue Nachbesserungen verlangt werden.

Die Piratenpartei bemerkt ,mit Sorge“, dass auch nach dem Scheitern von ACTA an internationalen Vereinbarungen über die Durchsetzung des Urheberrechts gearbeitet wird. ${ }^{168}$ Im Kern ist der Interessenkonflikt zwischen Urhebern und Nutzern ungelöst. Dass er nicht einfach nach dem Prinzip entschieden werden kann, die „Netzfreiheit“ genieße Vorrang, ist klar. Ebenso wenig wie die Auseinandersetzungen um Schmähungen und Mobbing im Netz allein zugunsten der Äußerungsfreiheit beendet werden können, ist das bei dem Konflikt um die InternetPiraterie möglich. Das Urheberrecht hat immateriellen und zugleich materiellen Gehalt, es verschafft Künstlern, Autoren und Komponisten Anerkennung und Entgeltansprüche (während das Persönlichkeitsrecht zunächst immaterielle Ansprüche

167 Opinion of European Academics on Anti-Counterfeiting Trade Agreement, zugänglich auf der Internetseite des Instituts für Rechtsinformatik der Leibniz Universität Hannover, vgl. http://www.iri.uni-hannover.de/acta-1668.html.

168 Pressemitteilung v. 15.10.2012 zu Verhandlungen über ein Comprehensive Economic and Trade Agreement (CETA), s. www.piratenpartei.de . S.a. die Hinweise von Küchemann 2012 auf weitere europäische und amerikanische Rechtsetzungsinitiativen. 
z.B. auf Unterlassung vermittelt und nur bei schweren Eingriffen auch Entschädigungen in Geld!). Das Recht derer, die geistige oder künstlerische Werke geschaffen haben, ist nicht weniger schutzwürdig als etwa die Gehaltsansprüche von Arbeitnehmern. Wie sollen sonst Künstler und Schriftsteller (und ihre Vermittler in Gestalt von Verlegern und Musikproduzenten) von ihrer Arbeit leben? Allein von dem Respekt anderer vor der eigenen Persönlichkeit kann niemand leben. Es ist also nicht einzusehen, dass die Leistungen der Journalisten, Schriftsteller und Künstler, die im Netz ausgestellt werden, nicht bezahlt werden sollen. Tatsächlich werden sie zum Teil ja über Werbeeinnahmen finanziert, so wie manche regionalen Wochenblätter, die unerbeten in die Briefkästen gesteckt werden, durch Anzeigen finanziert sind. Qualitätsjournalismus hat einen höheren Preis, und feste Abonnements sind eine solidere Basis als die schwankenden Reklameeinnahmen.

\section{Alternative Regelungsmodelle}

Da der Einzelne seine Vergütungsansprüche nicht allein durchsetzen kann, pflegt er die Ausübung seines Rechts einer Organisation, einem Verlag, Vertrieb oder einer Verwertungsgesellschaft (wie GEMA, VG Wort oder VG Kunst und Bild) zu übertragen, die dann einen Anteil an dem Entgelt erhält. Das erregt bei den Verfechtern der Unentgeltlichkeit Anstoß; sie halten diese Vermittler für Schmarotzer, die ohne eigene Leistung die Autoren und die Nutzer berauben. In manchen Fällen mag das zutreffen, in vielen anderen aber nicht; denn Verlage und Vertriebsfirmen müssen sehr wohl Geld aufwenden, um die künstlerischen und literarischen Leistungen zu vermarkten. Die rechtspolitische Aufgabe besteht darin, das Verhältnis zwischen Autoren und Vermittlern so zu regeln, dass beide Seiten zu angemessenen Erlösen kommen.

Diese Aufgabe ist nicht neu - es gibt ja ein ausgefeiltes Urheber- und Verlagsrecht und immer wieder Verbesserungen daran, und alle Seiten sind sich darin einig, dass die neuen Möglichkeiten der technischen Verbreitung von Inhalten zu neuen rechtlichen Gestaltungsformen führen müssen. Die Vergütungspflicht der Nutzer braucht auch nicht unbedingt an die einzelne Nutzung durch bestimmte Personen bzw. mittels bestimmter Computer anzuknüpfen. Es gibt bereits die Geräteabgabe: das (in gewissem Maße erlaubte) Kopieren von Texten und Bildern wird durch eine pauschale Abgabe vergütet, die der Hersteller, Händler oder Importeur von Kopiergeräten und Speichermedien oder der Betreiber von Kopiergeräten zahlen muss. ${ }^{169}$ 
Das Herunterladen von Texten oder Musik aus dem Internet ist technisch nicht dasselbe wie das „Ablichten“170 aus Büchern oder das Abspielen von CDs, und es ist schwieriger, einen passenden Ansatz für die Vergütung von Downloads zu finden. Aber mit einiger juristischer Kreativität" "171 müsste ein Interessenausgleich auch auf diesem Felde möglich sein. Inzwischen haben sich darum schon viele Experten bemüht. So hat die Enquete-Kommission „Internet und digitale Gesellschaft" des Deutschen Bundestages die in Betracht kommenden Vergütungsmodelle sorgfältig untersucht - ob es Werbefinanzierung, Flatrates oder Abonnements seien und an welche Nutzungsform sie zweckmäßig anknüpfen sollten. Die Empfehlungen waren freilich auch in der Kommission teilweise umstritten. ${ }^{172}$ Jedenfalls wäre eine allgemeine „Kulturabgabe“, die von jedem Internetnutzer zu entrichten wäre, zumindest solange ungerecht, wie das Internet noch so unterschiedlich genutzt wird wie derzeit. ${ }^{173}$

Verschiedene Forschungsgruppen und Arbeitskreise sind ebenfalls mit detaillierten Erörterungen befasst, und die Öffentlichkeit verfolgt diese Debatten. Wenn dies alles zu Ergebnissen führt, die - vielleicht als alternative Entscheidungsvorschläge - zur Abstimmung gestellt werden können, ist ein wichtiger Schritt getan. Langfristig wird sich das Urheberrecht sicher noch vielfach ändern, und auch das kann einen Fortschritt bedeuten.

Freiheit vom Staat und Schutz durch den Staat

\section{Grundrechtskonflikte und Interessenabwägungen}

Wie auf allen Gebieten des Zusammenlebens, gelten auch für das Verhalten im Netz die Grundrechte des Einzelnen und der privaten Vereinigungen, ohne dass dies besonders festgelegt werden müsste. Für die Sphäre des Geschäftlichen sind die Grundrechte der Vertragsfreiheit, der Berufs- und Gewerbefreiheit, der Koalitionsfreiheit und des Eigentums von besonderer Bedeutung. Die unternehmerische Freiheit darf aus Gründen des Gemeinwohls eingeschränkt werden. Das Bundesverfassungsgericht hat diese Grundaussage in vielen Details ausgeformt und die Interessen der Unternehmer und der Allgemeinheit dabei sorgfältig austariert. „Eigentum verpflichtet“; ,,sein Gebrauch soll zugleich dem Wohle der Allgemeinheit

170 Das Gesetz spricht altmodisch von „Ablichtungsgeräten“.

171 Vgl. a. die Hinweise bei Güntner 2012.

172 Dritter Zwischenbericht der Enquete-Kommission, Bundestags-Drs. 17/7899 v. 23.11 .2011 , insbes. S. $45 \mathrm{ff}$.

173 So auch der Arbeitskreis Urheberrecht der SPD-Bundestagsfraktion, Positionspapier v. 21.5.2012: „Zwölf Thesen für ein faires und zeitgemäßes Urheberrecht“. Dazu u.a. ein Interview mit einem Fachjournalisten: www.thomas-steiger.com . 
dienen“ (Art. 14 Abs. 2 GG). Die geistigen Freiheiten dürfen nur zugunsten höherrangiger Rechtsgüter eingeschränkt werden; eine Zensur ist ganz verboten. Das habe ich schon ausgeführt. ${ }^{174}$

Die Grundrechte schützen die Aktiven und die Passiven, je nach Bereich, und sie verpflichten den Staat sogar zum Schutz von Rechtspositionen der Individuen (z.B. gegen rechtswidrige Angriffe). Das Bundesverfassungsgericht hat dazu im Laufe der Zeit eine Lehre von den Schutzpflichten des Staates erarbeitet. In vielen Beziehungen stoßen Rechte und Interessen des einen auf solche eines anderen; zunächst können sich beide Seiten auf Grundrechtsartikel berufen. Der eine braucht Daten als „Rohstoff“ für seine geschäftlichen Aktivitäten, der andere will die Daten geheim halten, um seine Privatsphäre zu schützen oder wirtschaftliche Vorteile zu behalten, die ihm sein Wissensvorsprung bietet. Mit einer einseitigen Stellungnahme ist ein solcher Konflikt nicht zu befrieden. Deshalb müssen der Gesetzgeber und in zweiter Linie Verwaltung und Gerichte immer wieder Abwägungen vornehmen.

Was wäre, wenn der Staat sich überhaupt nicht mit all dem befassen würde, was im Internet geschieht? Die Vision der vollkommenen Staatsfreiheit muss jeden, der diesen Gedanken weiterdenkt, schaudern lassen. In den USA versuchen die Tea Party und große Teile der Republikaner gerade, den Staat handlungsunfähig zu machen. Die sozialen Folgen für die Mehrheit der Menschen sind erschreckend; noch erschreckender aber ist das Unvermögen der Menschen zu erkennen, dass sie immer mehr der Raffgier und Menschenverachtung derer ausgeliefert werden, die den „Markt“ steuern. Die Investoren, Finanziers und Manager, die nur den Gesetzen der Profitmaximierung folgen, gewinnen immer größere Freiheit und zahlen immer weniger Steuern, während die Masse der Arbeiter und Angestellten immer abhängiger wird. In Europa herrscht zum Glück bisher eine andere Vorstellung vom Verhältnis der Menschen zum Staat, aber die Bereitschaft, gemeinsame Angelegenheiten auch gemeinsam, also durch die kollektive Organisation Staat regeln zu lassen, nimmt auch hier ab. Der hochgelobte Philosoph Peter Sloterdijk propagiert die Abschaffung der Steuern zugunsten freiwilliger Gaben aller Wohlhabenden an die Allgemeinheit, ${ }^{175}$ und manche Vordenker der „Piraten“ erklären alle Staaten und alle Politiker zu Feinden des Volkes, Verschwörern gegen das Allgemeinwohl.

Freundlich ausgedrückt, sind viele Verteidiger der totalen Internet-Freiheit liebenswerte Idealisten, die an das Gute im Menschen glauben und die dunklen Seiten der menschlichen Seele nicht wahrnehmen mögen. Wer sich stets an die Regeln hält, die das Zusammenleben in der Gemeinschaft erträglich machen, wird auch bei der Internet-Nutzung niemandem Schaden zufügen. Dass aber die Menschen, 
die für den Staat handeln, stets böse Absichten - Unterdrückung, Korruption, Machtmissbrauch - hätten, passt nicht zu dieser gedanklichen Basis der Menschenfreundlichkeit. Gewiss, die Funktionsträger der Allgemeinheit verwalten Macht, und Macht kann korrumpieren - aber dagegen gibt es Sicherungen, die in aller Regel wirksam sind, nicht zuletzt die Kontrolle durch Medien und Öffentlichkeit. Und die schroffe Gegenüberstellung von „gutem“ Volk und „bösen“ Politikern ist schon deshalb unerträglich naiv, weil sie übersieht, dass ,gut“ und „böse" wie Machtbesitz und Machtmissbrauch auf beiden Seiten vorkommen, in der privaten Gesellschaft und bei staatlichen Funktionsträgern.

Eine Politik, die die Freiheit der Einzelnen sichern will, ist auf den Staat angewiesen. Zwar können zivilgesellschaftliche Aktivitäten den Staat entlasten; viele Probleme würden gar nicht entstehen, wenn die Bürgerinnen und Bürger die Steine des Anstoßes selbst aus dem Wege räumten. Das geschieht aber nicht ausreichend und oft eben nicht in der richtigen Weise, und so muss der Staat als höchste Instanz der Gemeinschaft immer wieder korrigierend eingreifen.

Auch das Internet existiert nicht in einer absolut staatsfreien Welt. Der Wettbewerb stellt sich in der „realen“ Welt nicht von selbst her, sondern muss vielfach erst geschaffen und immer wieder gefördert werden. Nicht anders ist es im Netz. Schon jetzt bilden die großen Vier Apple, Google, Amazon und Facebook ein Oligopol ${ }^{176}$, und es ist nicht garantiert, dass die Netzwirtschaft so aufgeteilt bleibt wie sie gegenwärtig ist. Die Regulierung funktioniert international noch nicht effektiv genug, aber ohne staatliche Regulierung entsteht auf die Dauer Wildwuchs, was mit Sicherheit nicht den Individuen nützt. Auch Datenschutz, wie ihn die Netznutzer ja nachdrücklich wünschen, entsteht nicht staatsfrei. Selbst da, wo Unternehmen sich ohne behördliche Aufforderung zu Datenschutz und Datensicherung verpflichten, geschieht dies vor dem Hintergrund der Rechtsnormen, die ein entsprechendes Verhalten verlangen - Selbstverpflichtung ,im Schatten des Rechts“.

Theoretisch wäre es sogar denkbar, das Netz einer staatlichen oder internationalen bzw. supranationalen (EU-) Kontrolle zu unterstellen, die Betreiber also als öffentliche, nicht gewinnorientierte Unternehmen zu errichten. (Ob das zu besseren Ergebnissen führen würde als die gegenwärtige privatwirtschaftliche Ordnung, mag dahinstehen.) Eine Rechtspflicht, die grundlegenden Dienstleistungen der Telekommunikation allen Einwohnern zur Verfügung zu stellen, kann einem marktbeherrschenden Unternehmen nach deutschem Recht schon auferlegt werden, seit das Fernmeldewesen im Jahre 1994 privatisiert wurde. Zu dieser „Universaldienstleistung" ist gegenwärtig die Deutsche Telekom AG verpflichtet. An weitergehende Einflussnahme des Staates denkt zur Zeit niemand; die politischen Widerstände dagegen wären enorm. 
Über die Sicherung der Individualrechte hinaus müssen wir uns fragen: Wie kann das Allgemeininteresse gegen Fehlentwicklungen geschützt werden? Die Literatur zum Internet hat uns die Augen dafür geöffnet, dass auch Werte der Allgemeinheit gefährdet sind, wenn wir uns der Internet-Faszination unkritisch hingeben. Der „Cyber-Utopismus“ hat sich auch nach Ansicht prominenter Netz-Aktivisten als Enttäuschung erwiesen; sie sprechen vom „Netzwahn“177 oder vom „kybernetischen Totalitarismus"178 und setzen ihm einen „digitalen Humanismus" entgegen. ${ }^{179}$ Den gilt es auszugestalten, und dazu gehört die „Verbesserung“ der Demokratie, die Entwicklung einer aufgeklärten Medienkompetenz, der Schutz gegen Meinungsmonopole und Konformismus, der Schutz von Kindern vor Verführung und Missbrauch. ${ }^{180}$ Die Verantwortung dafür, dass diese Ziele wirksam verfolgt werden, liegt beim Staat, nicht bei den Unternehmen. Die Wirtschaft ist aber als Teil der Gesellschaft sozialethisch in der Pflicht, den Boden dafür mit vorzubereiten und zu den notwendigen Maßnahmen das Ihre beizutragen.

\section{Exkurs: „Lernen im Netz“ statt ,, Schule vor Ort“?}

Wenn es nach den Ideen mancher Technikfreunde geht, werden auch Schulen und Hochschulen demnächst abgeschafft, weil das Lernen angeblich im virtuellen Raum besser organisiert werden kann als in den alten Gebäuden vor Ort. David Gelernter, der als Computerwissenschaftler in Yale lehrt und als jemand vorgestellt wird, der „die Grundlagen des World Wide Web“ geschaffen habe, ${ }^{181}$ meint offenbar ernsthaft: „Die Flut, die den größten Teil der heutigen Schulen und Universitäten hinwegschwemmen und uns stattdessen ein netzbasiertes Bildungssystem ohne Schulen bringen wird, hat bereits begonnen“. Ein solches Bildungssystem hätte nach seiner Meinung „,deutliche Vorzüge und hohe Kosten“. „Eltern, Schüler und Studenten werden aus einem weltweiten Bildungssystem auswählen können“; jeder kann in einem individuell angepassten Tempo lernen. Gelernter erkennt zwar an, dass „,man am besten lernt, wenn Lehrer und Schüler einander direkt gegenüberstehen“, aber diese Art des Lernens werde man ,weitgehend opfern“; denn die Großtechnologie werde sich gegen die Traditionen des Bildungswesens durchsetzen.

Hoffentlich nicht! Denn dieser Artikel ist ein Musterbeispiel dafür, auf welche Abwege jemand gerät, der eine politische und soziale Aufgabe allein von den technischen Möglichkeiten her lösen will, die auf dem Markt sind oder für die sich ein

177 Morozov 2011.

178 Lanier 2012, S. $30 \mathrm{ff}$.

179 Lanier 2012, S. 197 ff. S.a. die Rezension von H. Spiegel, FAZ v. 2.10.2010.

180 Zum Jugendschutz vgl. u.v.a. Ladeur 2000 S. 41 ff. (,Toleranz reicht nicht [immer] aus“).

181 So die FAZ in der Autorennotiz zu Gelernter 2012. 
Markt aufbauen lässt. Nicht einmal die höheren Kosten, die er selbst voraussagt, halten den Autor davon ab, seine Vorstellung von Online-Bildung zu verfolgen. „So denkt ein Technologe“, kommentiert mit Recht die FAZ-Redaktion und macht darauf aufmerksam, dass sich das Bildungswesen ,gegenüber technologischen Revolutionen bislang als äußerst träge erwiesen" hat. ${ }^{182}$ Bisher herrscht noch weitgehend Konsens darüber, dass die Schule ein sozialer Ort ist, an dem nicht nur Stoff gelernt, sondern auch soziales Verhalten geübt werden soll. Wie heißt es doch so schön: „Die Schule der Demokratie ist die Schule“. Die Online-Schule kann das nicht sein. Wer vorrangig auf das individuelle Lernen im und am Netz setzt, ${ }^{183}$ verkennt Grundeinsichten jeder Didaktik. Gewiss müssen wir das Schul- und Hochschulsystem immer wieder radikal kritisch überprüfen und dabei auch die Chancen des Lernens im Netz einkalkulieren. Aber es ist unwissenschaftlich und anmaßend, das Bildungswesen und die Lehrer (in aller Welt!) pauschal zu disqualifizieren und allein von der Technik her ein ideales neues System zu konzipieren.

Das Netz und die Netze: Neutralität, Kapazität und Sicherheit der Datentechnik

\section{Was bedeutet Netzneutralität?}

Zur „Freiheit des Internet“ gehört nach Ansicht von „Piraten“ und anderen NetzBegeisterten das Prinzip der Netzneutralität. ${ }^{184}$ Darunter versteht man, dass alle über das Netz zu transportierenden Datenpakete gleich behandelt werden, also weder eine Priorisierung nach Inhalten noch nach Art der Daten erlaubt ist. Wer zuerst kommt, wird zuerst bedient; in der Sprache der Informatiker: „Best-Effort-Prinzip“ und „First-In/First-Out-Prinzip“. „Bei Übertragungsengpässen entscheidet allein die zeitliche Reihenfolge der anfallenden Transportvorgänge darüber, welcher Vorgang zuerst abgewickelt wird“. ${ }^{185}$ Das ist zweifellos ein angemessener Grundsatz, solange die Engpässe allenfalls vorübergehender Natur sind und die Kapazitäten ständig erweitert werden, und überdies ein Prinzip, das die Gleichheit der Individuen betont und damit auch demokratiefreundlich zu sein scheint. Es bestärkt anscheinend auch den lakonischen Satz des Grundgesetzes: „Eine Zensur findet nicht statt“"(Art. 5 Abs. 1 Satz 3 GG).

182 Ebd. (,kau“).

183 So auch Beckedahl/Lüke 2012, 79 ff. Besonders irreal ist deren Vorstellung, die Studierenden würden künftig mit ihren Laptops jederzeit „gegenprüfen“, ob der Dozent richtig zitiert und , auf dem aktuellen Stand der Forschung“ ist. Das Abrufen von Informationen aus dem Netz ist noch lange kein Lernen und schon gar nicht Anleitung zu kritischem Denken.

184 Vgl. dazu die Beiträge in Kloepfer 2011.

185 Eberle 2011, S. 979 ff. (980) (m.w.N.). 
Aber das so verstandene Prinzip der Netzneutralität ist nicht selbstverständlich und wahrscheinlich nicht auf Dauer durchhaltbar. Je mehr Datentransporte auf das Internet verlagert werden, desto mehr Engpässe wird es geben. Längst werden ja nicht mehr überwiegend E-Mails verschickt und individuelle Bestellungen abgewickelt. Gewaltige Kapazitäten sind erforderlich, um die massenhafte Auftragsdatenverarbeitung, die Internet-Telefonie, Software-Downloads, Online-Spiele und Videodarbietungen zu ermöglichen. Das Livestreaming, die zeitgleiche Verbreitung von Fernsehprogrammen, ist überaus aufwendig, ebenso das zeitunabhängige On-Demand-Streaming etwa aus Mediatheken. Global vernetzte Produktionsprozesse sollen ebenso zuverlässig abgewickelt werden wie komplizierte Operationen, die internet-gestützt von Ärzten in weit entfernten Kliniken kooperativ durchgeführt werden.

Niemand hätte wohl etwas dagegen einzuwenden, wenn zwischen dem Angebot von Online-Spielen und der Übertragung von Operationsdaten unterschieden würde. Notrufe, Gesundheitsdienstleistungen und Katastrophenhilfe sind allemal dringender als das Angebot von Unterhaltungsprogrammen. Der wirtschaftlichen Logik entspräche es ohnehin, wenn unterschiedlich wichtige Datentransporte auch unterschiedlich bezahlt werden müssten - wir sind ja auch bereit, für eingeschriebene Sendungen ein Extraentgelt zu zahlen. Einer bevorzugten Behandlung beim Internettransport entspricht es, dass wir Briefe und Pakete per „Eilboten“ zustellen lassen - selbstverständlich gegen Sondergebühr. Als verbotene Priorisierung gilt nach dem Netzneutralitätsprinzip schon, dass eine Sendung um Bruchteile von Sekunden verzögert transportiert wird. Für die Qualität der Bild- und Tonübertragung kann das schon eine Rolle spielen. Aber Überlegungen, unterschiedliche Qualitätsstufen (-klassen) einzuführen, sind in der Welt. Einige Unternehmen wollen sich offenbar die bessere Qualität der Übertragung zusätzlich bezahlen lassen. Andere halten dagegen, insbesondere die Fernseh- und Videoanbieter, die an der höchstqualifizierten Transportmethode interessiert sind.

Sollten die Internetdienste eines Tages tatsächlich nach Maßgabe der „Quality of Services“ differenziert werden, wird auch die Frage wieder aufgeworfen, ob nicht damit der Zensur das Tor geöffnet wird. Das verfassungsrechtliche Zensurverbot richtet sich zwar nur an den Staat und nicht an private Internetunternehmen, aber die Freiheit der Kommunikation muss auch gegen die Macht der Internetbetreiber verteidigt werden. In der Aufstellung eines differenzierten Preiskatalogs für unterschiedliche Arten von Datentransport läge freilich noch kein Verstoß gegen das Zensurverbot; es muss nur sichergestellt werden, dass eine eventuelle Staffelung nicht nach den Inhalten der Datenpakete, sondern nach der Art und dem Zweck der Datentransporte vorgenommen wird - möglichst nach technischen Merkmalen, nach denen unterschiedliche Inhalte gleich behandelt werden. Möglicherweise wird eine zusätzliche Kennzeichnung der Pakete nötig sein: individuelle private 
Sendungen (auch an eine Mehrzahl von Empfängern) könnten anders markiert werden als Massendatenpakete zur Weiterverarbeitung, Fernseh- und Videosignale würden eine eigene Datenklasse bilden, und falls auch innerhalb dieser Klasse eine vorrangige Behandlung nötig werden sollte - z.B. für Notfälle wie die fernsehtechnische Assistenz beim Katastrophenschutz und anderen Hilfseinsätzen -, müssten Ausnahmen dafür durch entsprechende technische Qualifizierung ermöglicht werden. Natürlich ist das keine leichte Aufgabe, aber eine lösbare.

Die Internet-Nutzer hätten verständlicherweise die Sorge, dass die Betreiber ihre Leistungen zu teuer verkaufen. Diese stehen aber unter der Aufsicht der Kartellbehörden und der Gerichte, die auch auf diesem Feld eventuellen Auswüchsen mit den Instrumenten des Verbraucherschutzes begegnen können. Die Bundesnetzagentur als zuständige Sonderkartellbehörde für das Netz hat gesetzliche Befugnisse, die sie in diesem Fall aktivieren könnte. ${ }^{186}$

\section{Die Störanfälligkeit des Netzes}

Unsere vernetzte Welt wird ,immer störanfälliger““ ${ }^{187}$ Wenn eines Tages das Internet zusammenbricht, wird es in Teilen der Welt zappenduster, weil die Elektrizitätswerke ausfallen, und überall bleiben Bahnen, Fließbänder und Aufzüge stehen, kurz: alle Anlagen werden funktionsunfähig, die mit externen Computern verbunden sind. Wirtschaft und Verwaltung sind so sehr vom Netz abhängig, dass ein großer Teil der normalen Geschäftstätigkeit nicht mehr ohne dieses Instrument bewerkstelligt werden kann. Sabotageakte und Hackerangriffe machen schon jetzt vielen Unternehmen und Behörden schwer zu schaffen, und als Außenstehender wundert man sich, dass dieses Risiko von den Betreibern gering eingeschätzt wird. Als mögliche Ursachen sind aber auch Stromstörungen, Schlampereien, Überlastung und Fehlschaltungen zu bedenken, ${ }^{188}$ und die Folgen könnten für große Teile von Wirtschaft und Verwaltung verheerend sein.

Es gibt keinen zuverlässigen Schutz gegen eine solche Katastrophe. Es ist leicht, sich darüber zu mokieren, dass die zur Kontrolle der Informations- und Kommunikationstechnik eingesetzten Behörden personell schwach sind. ${ }^{189}$ Die Verantwortung für das Funktionieren des Internets liegt bei denen, die es aufgebaut haben, betreiben und nutzen, und die Behörden dürfen erst eingreifen, wenn gegen Rechtsnormen verstoßen wird. Gleichzeitig machen sich die hochgelobten Hacker - üb-

186 Vgl. Schwarz-Schilling 2011, S. 133 ff. Zu den europarechtlichen Bindungen auf diesem Gebiet: Mayer 2011, S. $81 \mathrm{ff}$.

187 Fischermann/ Hamann 2012.

188 Über frühere Zwischenfälle und Pannen im Netz berichten anschaulich z.B. Fischermann/ Hamann 2012, z.B. S. 13-15.

189 So etwa Fischermann/Hamann 2012, S. 202 ff. 
rigens auch sie nur eine kleine Schar von Menschen - über die Behörden lustig und werden dafür von manchen Medien noch belobigt. Dem Staat mangelnde Vorsorge gegen Störungen und Pannen vorzuwerfen, ist also allzu billig, zumal wenn gleichzeitig behauptet wird, die Behörden wüssten zu viel über die Menschen. Der Staat weiß eben nicht alles, und wir wollen es so.

Ein Patentrezept hat offenbar bisher niemand. Man hofft, dass die Katastrophe nicht eintreten oder dass sie nur andere treffen werde; bestenfalls werden Notaggregate vorgehalten. Sicherheit gegen Angriffe oder gegen Stromausfälle und Kabelzerstörung scheint es nicht zu geben. Wer insofern auf den Staat oder die Staatengemeinschaft hofft, macht sich Illusionen. Die privaten Betreiberunternehmen haben ein starkes eigenes Interesse daran, dass das Netz leistungsfähig bleibt, und sie tun viel für die Sicherheit ihrer Anlagen. Der Staat könnte das wohl kaum besser, obwohl er kein Profitinteresse hätte und daher (vielleicht) niedrigere Kosten verursachen würde.

\section{Das Netz und die Netze}

Eine Chance, etwas mehr Funktionssicherheit der Datenverarbeitung und der elektronischen Kommunikation zu gewinnen, besteht darin, das eine große Netz in verschiedene, voneinander getrennte Netze aufzuteilen. In manchen Bereichen könnte ganz auf die Vernetzung verzichtet werden. Isolierte Systeme sind besser gegen Eingriffe von außen und Netzstörungen geschützt. So nahe diese Lösung auch liegt, sie ist bisher nur in bescheidenen Ansätzen und offenbar halbherzig verwirklicht.

Die WikiLeaks-Enthüllungen haben offengelegt, dass sogar die amerikanische Armee und das US-Außenministerium viel zu viele Zugriffsmöglichkeiten auf ihre internen Nachrichtennetze zugelassen oder ermöglicht haben. Dass andererseits auch solche Dateien, die nur für einen kleinen Kreis von Personen von Interesse sind, im weltweiten Netz gespeichert werden, war schon immer fragwürdig. Warum müssen Dozenten und Studenten einer deutschen Universität miteinander über Server in Amerika kommunizieren? Warum werden die Programmdaten eines Stadttheaters und der Fahrplan eines örtlichen Verkehrsunternehmens durch Überseekabel oder Satelliten um die Welt gelenkt, ehe sie bei den Interessenten „nebenan" eintreffen? Ist es nötig, dass meine Verbrauchsdaten, die nur für den regionalen Stromversorger bestimmt sind, über das Internet transportiert werden? Führt die ökonomische Logik zwingend zur Verarbeitung von Datenmassen aus aller Welt bei beliebig entfernten Rechenzentren?

Denken wir daran, dass auch höchst gefährliche und gefährdete industrielle Anlagen, Atomkraftwerke und das Stromnetz ebenso wie Verkehrsleitanlagen und die 
Flugsicherung durch Computer gesteuert werden, die an das Internet angeschlossen sind, so kann uns angst und bange werden. „Solche kritischen Infrastrukturen, die wir für unseren Alltag dringend brauchen, bei denen es um Leben und Tod geht sie müssen unwiderruflich vom Netz". 190

Aber auch sonst liegt es nahe, das einheitliche World Wide Web von vielen Anwendungen zu entlasten, die nur lokale oder regionale Bedeutung haben, und diese in eigene, speziell zweckbestimmte Netze zu überführen. ${ }^{191}$ Damit ist nicht gemeint, dass das Netz vollständig zerlegt, vor allem nicht dass es in die Hände der nationalen Regierungen gelegt werden soll, die dann ihr Territorium ,perfekt“ von dem weltweiten Netz abkoppeln könnten. Wir dürfen die große Errungenschaft eines globalen Informations- und Kommunikationsmediums ohne nationale Grenzen nicht aufgeben. Eine - immerhin denkbare - Vorschrift der Europäischen Union, dass die Daten über Europäer nur in Europa gespeichert werden dürfen, ${ }^{192}$ wäre ebenfalls ein Rückschritt. Aber eine nach Zwecken und Teilnehmerkreisen differenzierte Datenverarbeitung könnte durchaus freiheitsfreundlich gestaltet werden - ginge es doch auch um eine Form von, ,informationeller Gewaltenteilung“. Dieses seit den Anfängen des Datenschutzes bekannte Prinzip wird oft vernachlässigt.

Der freie Zugang zu allen großen und kleinen Wissens-Portalen und Diskussionsforen könnte dabei ohne weiteres gewährleistet bleiben, solange eben nicht nationale Regierungen die Herrschaft darüber gewinnen. Die Sicherheit und Zuverlässigkeit der Systeme aber würde deutlich erhöht, und damit würde vielen Ängsten der Boden entzogen, die in unserer Gesellschaft herrschen.

190 Fischermann/Hamann 2012, S. 246.

191 So auch Fischermann/Hamann 2012, S. 243 ff.

192 Als Denkmodell bei Fischermann/Hamann 2012, S. 243. 